\title{
Prevalence and potential fitness cost of weak phosphine resistance in Tribolium castaneum (Herbst) in eastern Australia
}

\author{
Gregory J. Daglish a, b, *, Manoj K. Nayak ${ }^{\text {a, b }}$, Hervoika Pavic a, b, Lawrence W. Smith b, c \\ a Department of Agriculture, Fisheries and Forestry, Queensland, Ecosciences Precinct, GPO Box 267, Brisbane, QLD 4001, Australia \\ ${ }^{\mathrm{b}}$ Plant Biosecurity Cooperative Research Centre, GPO Box 5012, Bruce, ACT 2617, Australia \\ ${ }^{\mathrm{c}}$ Department of Agriculture, Fisheries and Forestry, Queensland, Leslie Research Centre, PO Box 2282, Toowoomba, QLD 4350, Australia
}

\section{A R T I C L E I N F O}

\section{Article history:}

Received 21 August 2014

Received in revised form

20 November 2014

Accepted 22 November 2014

Available online 8 December 2014

\section{Keywords:}

Fumigation

Resistance

Stored grain beetle

\begin{abstract}
A B S T R A C T
The prevalence of resistance to phosphine in the rust-red flour beetle, Tribolium castaneum, from eastern Australia was investigated, as well as the potential fitness cost of this type of resistance. Discriminating dose tests on 115 population samples collected from farms from 2006 to 2010 showed that populations containing insects with the weakly resistant phenotype are common in eastern Australia $(65.2 \%$ of samples), although the frequency of resistant phenotypes within samples was typically low (median of 2.3\%). The population cage approach was used to investigate the possibility that carrying the alleles for weak resistance incurs a fitness cost. Hybridized populations were initiated using a resistant strain and either of two different susceptible strains. There was no evidence of a fitness cost based on the frequency of susceptible phenotypes in hybridized populations that were reared for seven generations without exposure to phosphine. This suggests that resistant alleles will tend to persist in field populations that have undergone selection even if selection pressure is removed. The prevalence of resistance is a warning that this species has been subject to considerable selection pressure and that effective resistance management practices are needed to address this problem. The resistance prevalence data also provide a basis against which to measure management success.
\end{abstract}

Crown Copyright $\odot 2014$ Published by Elsevier Ltd. All rights reserved.

\section{Introduction}

Phosphine fumigation is commonly used to control the rust-red flour beetle, Tribolium castaneum (Herbst), which is one of the most important pests of stored cereals. However, effective management of this pest is threatened by phosphine resistance which has been reported from many parts of the world (e.g. Champ and Dyte, 1976; Price and Mills, 1988; Herron, 1990; White and Lambkin, 1990; Rajendran, 1994; Benhalima et al., 2004; Pimentel et al., 2007; Opit et al., 2012). Studies on the inheritance of phosphine resistance in $T$. castaneum show that there are two broad levels of resistance (weak and strong) in this species, with two major genes (rph1 and rph2) controlling their expression (Bengston et al., 1999; Jagadeesan et al., 2012). Insects with weak resistance are homozygous for rph1 and insects with strong resistance are homozygous for both rph1 and rph2. These genes are incompletely recessive and

\footnotetext{
* Corresponding author. Department of Agriculture, Fisheries and Forestry, Queensland, Ecosciences Precinct, GPO Box 267, Brisbane, QLD 4001, Australia.

E-mail address: greg.daglish@daff.qld.gov.au (G.J. Daglish).
}

not sex-linked. Weakly resistant strains have been reported to be up to 12.7 times more resistant than susceptible strains, and a strongly resistant strain over 431 times more resistant, based on adult mortality after a 20-h exposure to phosphine (Bengston et al., 1999; Jagadeesan et al., 2012).

There is very limited information on the prevalence of phosphine resistance in $T$. castaneum in eastern Australia. Two studies from the 1980s show that the frequency of field populations of T. castaneum with phosphine resistant insects was low in eastern Australia at that time. White and Lambkin (1990) found seven out of 36 samples (19\%) collected from across Queensland from 1986 to 1988 had survivors at the FAO discriminating dose of $0.03 \mathrm{mg} \mathrm{L}^{-1}$ for $20 \mathrm{~h}$ (FAO, 1975) but none had survivors at $0.04 \mathrm{mg} \mathrm{L}^{-1}$. In another study, using $0.05 \mathrm{mg} \mathrm{L}^{-1}$ for $20 \mathrm{~h}$, Herron (1990) found that there were survivors in five out of 23 samples (22\%) collected from the Edgeroi district in northern New South Wales in 1985, and four out of 83 samples (5\%) collected from the Grong Grong district in southern New South Wales in 1986. After several decades of phosphine use there is need for more recent information on the prevalence of phosphine resistance in $T$. castaneum, including information on both weak and strong resistance. 
Although resistant individuals clearly have an advantage over susceptible individuals in the presence of a toxicant, it is commonly assumed that resistant individuals are less fit than their susceptible conspecifics in the absence of selection pressure, because resistance is rare before selection is imposed by humans (McKenzie, 1996). Therefore, knowing if there are fitness costs associated with phosphine resistance could help in resistance management. Fitness has been investigated in a range of insect pests of stored grain using different experimental approaches (Pimentel et al., 2007; Schlipalius et al., 2008; Sousa et al., 2009; Pimentel et al., 2011; Jagadeesan et al., 2012, 2013; Daglish et al., 2014). Some studies have used the population cage approach in which susceptible and resistant strains are hybridized and the resulting populations are reared in the absence of phosphine selection. Changes in the frequency of resistant phenotypes or resistant alleles are then determined. Others have examined a range of population samples with the aim of correlating physiological or ecological parameters with the strength of resistance determined through bioassays. Overall, there has been no consistent conclusion about whether being resistant has a fitness cost. In the case of $T$. castaneum, Pimentel et al. (2007) found that reproduction and respiration rate were negatively correlated with resistance ratio across a range of field samples, and Sousa et al. (2009) reported that more resistant samples tended to have slower development than more susceptible strains. These studies provide strong evidence for fitness costs associated with phosphine resistance, but they used unselected field samples which may have contained multiple genotypes. As mentioned earlier, there are two broad levels of resistance (weak and strong) controlled by two major genes, so it is desirable to understand fitness consequences of being weakly or strongly resistant to phosphine. Recently, Jagadeesan et al. (2012) reported that there was no evidence of fitness costs associated with either weak or strong resistance in $T$. castaneum, using the population cage approach and phenotype testing. The study used a single segregating population each from the following crosses: susceptible $\times$ weakly resistant, weakly $\times$ strongly resistant cross and susceptible $\times$ strongly resistant. Subsequently, Jagadeesan et al. (2013) reported that molecular analysis of the susceptible $\times$ strongly resistant population showed an increase in the frequency of the rph 1 allele and a decrease in the frequency of the rph2 resistance allele over time indicating a fitness cost associated with that allele.

In this paper we report the results of a resistance survey showing the prevalence of weak and strong phosphine resistance in T. castaneum in eastern Australia, and a laboratory investigation into whether there is detectable fitness cost associated with weak resistance. The survey involved the collection and testing of more than 100 samples collected from farms in Queensland and northern New South Wales from 2006 to 2010. Fitness was investigated using the population cage approach, in which weakly resistant and susceptible strains were hybridized and phenotype frequency was estimated in segregating populations reared for seven generations in the absence of phosphine selection. The fitness study used one resistant strain and two different susceptible strains, in case genetic background of either of the susceptible strains used in the cross influenced the outcome.

\section{Materials and methods}

\subsection{Phosphine fumigation bioassays}

Bioassays followed the methods recommended by FAO (1975). A phosphine source was generated from an aluminium phosphide tablet and collected over acidified water. The source concentration was measured by gas chromatography using a gas density balance
(Aerograph Model 90-P; Varian, Mount Waverley, Victoria, Australia). The carrier gas was dichlorofluoromethane (Refrigerant F22; Lovelock Luke, Mayne, Queensland, Australia). Adults (1-3 wk old) were added to ventilated plastic soufflé cups which were then placed inside gas-tight desiccators, and gas-tight syringes were used to inject the required amount of phosphine through a septum in the lid of each desiccator. Adults were fumigated for $20 \mathrm{~h}$ at $25^{\circ} \mathrm{C}$ and $55 \%$ r.h., and then retained in a small quantity of whole wheat flour plus yeast $(10: 1)$ for 2 wk until end-point mortality was assessed.

\subsection{Prevalence of phosphine resistance in eastern Australia}

Prevalence of phosphine resistance was determined by discriminating dose tests on population samples collected from farms in Queensland and northern New South Wales from 2006 to 2010. All samples were cultured in a rearing medium of whole wheat flour plus yeast $(10: 1)$ at $30{ }^{\circ} \mathrm{C}$ and $55 \%$ r.h. and tests were conducted on the $\mathrm{F}_{1}$ generation at two discriminating doses. Only those samples that were started with at least 50 field adults were used, to minimize the potential of founder effects biasing test results. One dose was the FAO discriminating dose of $0.03 \mathrm{mg} \mathrm{L}^{-1}$ and the other was a higher dose of $0.25 \mathrm{mg} \mathrm{L}^{-1}$. For each population sample, three batches of at least 50 adults (1-3 weeks postemergence) were tested at $0.03 \mathrm{mg} \mathrm{L}^{-1}$, three batches were tested at $0.25 \mathrm{mg} \mathrm{L}^{-1}$ and a single batch of at least 30 adults served as an untreated control. These fumigations were undertaken as described earlier. Any samples with $>10 \%$ control mortality were excluded from further consideration.

Kendall's rank non-parametric correlation was used to test the possibility that the frequency of samples with survivors changed during the 5 years of sampling (GenStat, 2008). This test does not provide a regression equation or a functional relationship but tests whether the dependent variable increases or decreases monotonically as a function of the independent variable (Sokal and Rohlf, 1995). Median survival in samples was calculated and median absolute deviation was used as the measure of variation around the median. Median survival was assumed to represent the percentage of homozygous resistant individuals in a typical population sample from the surveyed area. The frequency of the resistant allele (p) was calculated as the square root of the frequency of the homozygous resistant individuals, assuming monogenic inheritance (see Results) and Hardy-Weinberg equilibrium (McKenzie, 1996). The frequency of susceptible allele (q) was calculated as $1-\mathrm{p}$, and the frequency of homozygous susceptible individuals was the calculated as $\mathrm{q}^{2}$. The frequency of heterozygotes was calculated as $1-(\mathrm{p}+\mathrm{q})$.

\subsection{Fitness of phosphine resistance}

Three laboratory strains of $T$. castaneum were used in this study: a phosphine resistant strain (QTC300) and two phosphine susceptible strains (QTC4 and QTC285). QTC4 and QTC300 were developed from adults collected from Queensland in 1965 and 1990 (Bengston et al., 1999) respectively. QTC285 was developed by synthesising a range of laboratory and field strains in 1985, and was characterised by having a weak resistance to organophosphorus insecticides and pyrethroids (Collins, 1990). Bengston et al. (1999) had reported that the resistant strain had a resistance ratio of 12.7 based on the $\mathrm{LC}_{50}$ value for adults fumigated for $20 \mathrm{~h}$. The fitness study was done several years after that study but this strain was re-tested at the time of the fitness study confirming its resistance level. These strains were cultured in a rearing medium of whole wheat flour plus yeast $(10: 1)$ at $30{ }^{\circ} \mathrm{C}$ and $55 \%$ r.h. until needed for experiments. 
In the first part of the study two test populations were produced by crossing the resistant QTC300 strain with the susceptible QTC4 strain (Resistant $\times$ Susceptible 1). Pupae were removed from rearing medium and placed individually into $30-\mathrm{ml}$ plastic soufflé cups with $10 \mathrm{~g}$ of rearing medium after sexing according to the method of Hinton (1942). After adult emergence single pairs of virgin adults were placed into rearing medium (10 g) for 7 days after which the pairs were removed and the cups stored until the adult progeny emerged. The $F_{1}$ progeny arising from any single pair matings (50-100 pairs) were pooled to begin each test population, and the resulting two populations were maintained without selection for seven non-overlapping generations. Every generation the frequency of susceptible phenotypes was determined by testing samples of adults with the discriminating dose of $0.04 \mathrm{mg} \mathrm{L}^{-1}$ using the FAO Method (FAO, 1975). This dose is not expected to kill resistant insects (Bengston et al., 1999). The second part of the study was identical to the first except that the resistant QTC300 strain was hybridized with the second susceptible QTC285 strain (Resistant $\times$ Susceptible 2). All crosses and rearing were conducted at $25{ }^{\circ} \mathrm{C}$ and $55 \%$ r.h., while phosphine testing was conducted at $25^{\circ} \mathrm{C}$ and $70 \%$ r.h.

There were two test populations resulting from each of the two crosses (i.e. Resistant $\times$ Susceptible 1 and Resistant $\times$ Susceptible 2 ). For each population, $400 \mathrm{~F}_{1}$ adults were divided into two lots of 200 and each lot was placed into a separate jar containing $400 \mathrm{~g}$ of rearing medium. Thus each population comprised two jars. After $1 \mathrm{wk}$ of oviposition the parental insects were removed and pooled for testing and the cultures were held stored for $7 \mathrm{wk}$ until the adult progeny were removed. The $\mathrm{F}_{2}$ progeny emerging from the two jars were pooled and 400 adults were used to set up two new jars as described above. This process was continued each generation until the $\mathrm{F}_{7}$ generation emerged.

The adults used to set up each new generation were subjected to the discriminating dose of $0.04 \mathrm{mg} \mathrm{L}^{-1}$ for $20 \mathrm{~h}$ using the methods described earlier, to estimate the frequency of phenotypically susceptible individuals. Three hundred adults used were fumigated and 50 served as controls. Adults were confined within ventilated polystyrene vials in batches of 50 inside gas-tight glass desiccators. Three desiccators were used: one for the control insects and two for the fumigated insects (i.e. 150 per desiccator). After $20 \mathrm{~h}$ exposure to phosphine the adults were retained in a small quantity of medium for 2 wk when end-point mortality was assessed.

Weak resistance in $T$. castaneum is conferred by one major gene that is almost completely recessive (Bengston et al., 1999; Jagadeesan et al., 2012). All $F_{1}$ individuals would have been heterozygotes but subsequent generations could have contained homozygous resistant individuals, homozygous susceptible individuals and heterozygous individuals. In theory, $25 \%$ of the $F_{2}$ should be homozygous resistant and survived the discriminating dose, but the percentage of survivors is likely to be less than this (see Discussion). If having a resistance gene confers no advantage or disadvantage in the absence of phosphine, then according to the Hardy-Weinberg equilibrium the proportion of each genotype should remain constant from the $\mathrm{F}_{2}$ generation onwards. Therefore, the $F_{1}$ generation was excluded from statistical analysis. A nonparametric test, Kendall's rank correlation coefficient, was used in lieu of regression analysis to test whether mean mortality was dependent on generation number (GenStat, 2008).

\section{Results}

\subsection{Prevalence of phosphine resistance in eastern Australia}

Data from resistance testing of 115 farm samples were analysed. Survivors were present in $65.2 \%$ of the farm samples tested at
$0.03 \mathrm{mg} \mathrm{L}^{-1}$, and survival in those samples was $0.3-27.1 \%$. These tests were conducted on the $F_{1}$ progeny of field populations collected from 2006 to 2010, and there was a weak positive correlation between percentage survival and date of testing $\left(\tau_{6}=0.2292, P=0.0001\right)$, indicating a small increase in the frequency of resistant populations from 2006 to 2010 (Fig. 1). The highest median survival was 2.4\% for samples tested in 2010.

Typically, there were few survivors in the resistant samples, with median survival of $2.3 \%$ (median absolute deviation $=1.6 \%$ ). There were no survivors in any of the farm samples at the higher discriminating dose of $0.25 \mathrm{mg} \mathrm{L}^{-1}$. We conclude that $T$. castaneum populations containing individuals of the weakly resistant phenotype are common in eastern Australia, but that resistant populations typically contain few resistant individuals. Although no farm samples had survivors at the higher discriminating dose, strong phosphine resistance was evident in one sample collected in 2007 from a large grain handling depot in southern Queensland. This sample was collected following concerns about fumigation failure, and contained only eight founding adults. For these reasons it cannot be considered an unbiased sample like the farm samples, but shows that this type of resistance is present.

The frequency of the weakly resistant allele should be $15.2 \%$ based on $2.3 \%$ of individuals in a typical population sample from the surveyed area being homozygous weakly resistant, and assuming monogenic inheritance and Hardy-Weinberg equilibrium. With an allele frequency of $15.2 \%$, frequencies of homozygous resistant, homozygous susceptible and heterozygous individuals should be $2.3,72.0$ and $25.7 \%$, respectively. This means that $28 \%$ of individuals should be carrying at least one copy of the resistant allele.

\subsection{Fitness of phosphine resistance}

Every generation from the $F_{1}$ to the $F_{7}$ was tested at the discriminating dose of $0.04 \mathrm{mg} \mathrm{L}^{-1}$ for $20 \mathrm{~h}$ (Table 1 ). In the case of the two populations started by crossing Resistant $\times$ Susceptible 1 , mortality of the $F_{1}$ was $100 \%$ and mortality for the $F_{2}-F_{7}$ generations was $83.1-96.0 \%$ and $92.3-98.0 \%$ for Populations $A$ and $B$ respectively. In the case of the two populations started by crossing Resistant $\times$ Susceptible 2, mortality of the $\mathrm{F}_{1}$ was $100 \%$ and mortality for the $F_{2}-F_{7}$ generations was $72.0-89.0 \%$ and $73.2-87.4 \%$ for Populations $C$ and D respectively. Kendall's rank correlation coefficient was calculated for the data for the $F_{2}-F_{7}$ generations and this failed to detect a significant correlation between mortality and generation in Populations A, B, C or D (Table 1). There was no detectable trend in the frequency of susceptible phenotypes,

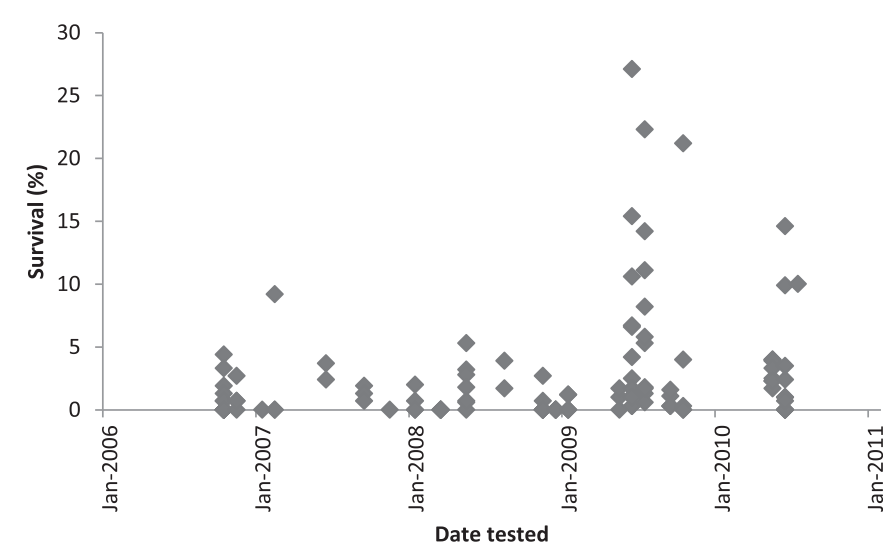

Fig. 1. Adult survival (\%) in samples of Tribolium castaneum from eastern Australia in phosphine bioassays ( $0.03 \mathrm{mg} \mathrm{L}^{-1}, 20 \mathrm{~h}$ exposure, $14 \mathrm{~d}$ post-fumigation assessment) in relation to date of testing. 
Table 1

End-point mortality (\%) (mean $\pm \mathrm{SD}, N=2$ ) of adults of Tribolium castaneum populations exposed to $0.04 \mathrm{mg} \mathrm{L}^{-1}$ for $20 \mathrm{~h}$ at $25^{\circ} \mathrm{C}$ and $70 \%$ r.h., where each population was started by crossing a weakly phosphine resistant strain with one of two susceptible strains.

\begin{tabular}{llllll}
\hline Generation $^{\mathrm{a}}$ & \multicolumn{2}{l}{$\begin{array}{l}\text { Resistant } \times \text { susceptible } 1 \\
(\mathrm{QTC3} 00 \times \text { QTC4 })\end{array}$} & & \multicolumn{2}{l}{$\begin{array}{l}\text { Resistant } \times \text { susceptible } 2 \\
(\text { QTC300 } \times \text { QTC285 })\end{array}$} \\
\cline { 2 - 3 } \cline { 5 - 6 } \cline { 5 - 6 } & Population A & Population B & & Population $\mathrm{C}$ & Population D \\
\hline $\mathrm{F}_{1}$ & $100 \pm 0.0$ & $100 \pm 0.0$ & & $100 \pm 0.0$ & $100 \pm 0.0$ \\
$\mathrm{~F}_{2}$ & $96.0 \pm 1.9$ & $93.8 \pm 1.5$ & & $89.0 \pm 1.4$ & $87.4 \pm 1.9$ \\
$\mathrm{~F}_{3}$ & $90.4 \pm 0.5$ & $92.3 \pm 0.5$ & & $82.9 \pm 1.4$ & $88.8 \pm 9.4$ \\
$\mathrm{~F}_{4}$ & $94.2 \pm 2.9$ & $95.7 \pm 1.5$ & & $72.0 \pm 6.6$ & $73.2 \pm 3.7$ \\
$\mathrm{~F}_{5}$ & $92.4 \pm 7.0$ & $96.4 \pm 0.5$ & & $82.3 \pm 2.8$ & $84.5 \pm 2.2$ \\
$\mathrm{~F}_{6}$ & $83.1 \pm 1.5$ & $93.7 \pm 0.4$ & & $85.9 \pm 0.5$ & $90.2 \pm 0.9$ \\
$\mathrm{~F}_{7}$ & $90.1 \pm 4.6$ & $98.0 \pm 1.0$ & & $87.4 \pm 3.6$ & $86.4 \pm 3.8$ \\
Mean $\mathrm{F}_{2}-\mathrm{F}_{7}$ & 91.0 & 95.0 & & 83.2 & 85.0 \\
\hline
\end{tabular}

${ }^{a}$ Kendall's rank correlation coefficient calculated for the data for the $F_{2}-F_{7}$ gen erations and detected no significant correlation in Population A $\left(\tau_{6}=-0.6000\right.$, $P=0.932)$, Population B $\left(\tau_{6}=0.4667, P=0.0681\right)$ or Populations C or D (with identical results: $\tau_{6}=0.0667, P=0.360$ ).

meaning that there was no evidence of a fitness cost associated with being resistant.

\section{Discussion}

Phosphine resistance is common in samples of $T$. castaneum collected from farms in eastern Australia, but the frequency of phenotypically resistant individuals is generally low. We calculated that $65.2 \%$ of samples collected from Queensland and northern New South Wales from 2006 to 2010 contained resistant individuals, and that the median frequency of resistant individuals in a field sample was $2.3 \%$. Two types of phosphine resistance, termed weak and strong resistance, are known to occur in $T$. castaneum (Jagadeesan et al., 2012), There was no evidence of strong phosphine resistance in any farm samples during the present survey, but one sample collected in 2007 from a large grain handling depot was diagnosed as strongly resistant. This was a targeted sample collected following concerns about fumigation failure, so it cannot be considered an unbiased sample like the farm samples. Strong resistance to phosphine has been known to exist in $T$. castaneum from eastern Australia for over a decade (Jagadeesan et al., 2012). Overall, however, the current results show that weak resistance is common in eastern Australia while strong resistance is rare. These results are similar to those recently published for the rice weevil, Sitophilus oryzae (L.), in eastern Australia (Daglish et al., 2014). Populations of S. oryzae with weak resistance were common but the frequency of phenotypically resistant individuals within populations was typically low. Resistant populations of $T$. castaneum seem to be more prevalent than they were in the 1980s, although direct comparisons with the earlier surveys of White and Lambkin (1990) and Herron (1990) are difficult because of differences in sampling approaches and testing between the studies. Weak resistance in T. castaneum is almost completely recessive (Bengston et al., 1999; Jagadeesan et al., 2012), and so heterozygotes are very unlikely to survive the FAO discriminating dose of $0.03 \mathrm{mg} \mathrm{L}^{-1}$ for $20 \mathrm{~h}$. Although the proportion of survivors in tests using this discriminating dose was typically low (2.3\%), potentially more individuals were carrying the resistance allele because heterozygotes are unlikely to survive. For example, if we assume that inheritance of weak resistance is monogenic, the segregating populations in the field having the three genotypes ( $r r, r s, s s)$, and that the genotypes are in Hardy-Weinberg equilibrium, then we estimated the frequency of individuals carrying at least one copy of the resistance allele to be $28 \%$. Genotype frequencies in the field may vary from
Hardy-Weinberg because of mutation, migration and genetic drift (McKenzie, 1996), and so our estimate is illustrative only.

The current study revealed no evidence that $T$. castaneum individuals carrying alleles for weak resistance were more or less fit than susceptible insects. This is based on the population cage approach (i.e. rearing without selection pressure for seven generations) and phenotype bioassays of adults from each generation. In population cage studies it is possible that genetic drift and bottlenecking may influence the outcome, or that the genetic background of the susceptible strain may have some influence on the results. The current study had two features which reduced the risk of the results being affected by these factors. First, the study populations were derived from crossing the weakly resistant strain with one or the other of two susceptible strains. Second, there were two replicate populations in each case. The finding of no evidence of a fitness cost associated with weak resistance in T. castaneum is similar to that obtained in a recently published study on T. castaneum using the population cage approach and phenotype testing of a single population derived by a susceptible $\times$ weakly resistant cross (Jagadeesan et al., 2012), although subsequent molecular screening of sub-samples from that study showed a slight increase in the frequency of the rph1 resistance allele over time (Jagadeesan et al., 2013). The finding of no apparent fitness cost is also similar to a recently published study on $S$. oryzae using the population cage approach and phenotype testing of replicated populations derived from a susceptible and weakly resistant cross (Daglish et al., 2014). On the basis of published population cage studies and the one reported in this paper, it appears that there is little or no cost or benefit associated with weak phosphine resistance.

The population cage approach is not the only one that has been used to investigate the potential for fitness costs associated with phosphine resistance. The population cage approach has the advantage that it may measure the net effect of resistance on multiple biological parameters, but resistance has the potential to affect a range of physiological and ecological parameters, and other approaches will be complementary, and used specifically when information on potential effects on specific traits are needed. Some researchers have investigated various biological parameters across a range of populations with a species, with varying levels of resistance measured using dose-response bioassays. Pimentel et al. (2007) found that reproduction and respiration rates were negatively correlated with resistance ratio across a range of field samples of T. castaneum. Sousa et al. (2009) reported a tendency for slower development in more resistant samples, when a range of T. castaneum samples were compared. These studies provide strong evidence for fitness costs associated with phosphine resistance, but they used unselected field samples which may have contained multiple genotypes. Knowledge of the potential fitness costs associated with phosphine resistance would benefit from studies on specific biological parameters but using strains of known genotype. Care would need to be taken to ensure that any costs were correctly attributed to being resistant and not to genetic differences between strains unrelated to resistance.

Weak phosphine resistance has been reported to be conferred by one major gene (Bengston et al., 1999; Jagadeesan et al., 2012). In the fitness study, therefore, $25 \%$ of the $F_{2}$ adults should have been homozygous resistant and survived the discriminating dose. In fact, survival was lower than this in all four experimental populations. In the studies of Bengston et al. (1999) and Jagadeesan et al. (2012), observed survival was less than $25 \%$ at doses where $25 \%$ of the $F_{2}$ adults were predicted to survive. Although monogenic inheritance was supported by the data in those studies, it is possible that one or more minor genes may also be involved, explaining discrepancy between predicted and observed survival. The results of the fitness 
study and those of the two genetic studies show that the results of phenotype testing using discriminating doses should be seen as indicative, while the results of this investigation of the prevalence of resistance should be seen as indicative and probably conservative.

The results of the studies reported in this paper provide insights into the prevalence of phosphine resistance in $T$. castaneum in eastern Australia and potential fitness costs associated with phosphine resistance. The fact that populations with weak resistance are common in eastern Australia, and apparently more common than in the 1980s, represents a warning as does the apparent absence of fitness cost associated with weak resistance. Despite the commonness of the weak resistance in eastern Australia, strong resistance in this region is rare. Effective resistance management practices are needed to combat resistance in this species, and future resistance surveys should be undertaken to monitor success in this regard.

\section{Acknowledgements}

With regard to the resistance prevalence research, the authors gratefully acknowledge the support of Plant Biosecurity Cooperative Research Centre (Project No: PBCRC3035), established and supported under the Australian Government's Cooperative Research Centres program (http://www.crcplantbiosecurity.com. au). The authors would also like to acknowledge the support of the Australian Centre for International Agricultural Research(Project No. 9415) with regard to the fitness research. We thank Megan Cameron, Alexandra Glauerdt and Linda Bond for laboratory technical assistance. We are also grateful to Rajaswaran Jagadeesan for critical comments on the manuscript.

\section{References}

Bengston, M., Collins, P.J., Daglish, G.J., Hallman, V.L., Kopittke, R.A., Pavic, H., 1999. Inheritance of phosphine resistance in Tribolium castaneum (Coleoptera: Tenebrionidae). J. Econ. Entomol. 92, 17-20.

Benhalima, H., Chaudhry, M.Q., Mills, K.A., Price, N.R., 2004. Phosphine resistance in stored-product insects collected from various grain storage facilities in Morocco. J. Stored Prod. Res. 40, 241-249.

Champ, B.R., Dyte, C.E., 1976. Report of the FAO Global Survey of Pesticide Susceptibility of Stored Grain Pests. Food and Agriculture Organisation, Rome.
Collins, P.J., 1990. A new resistance to pyrethroids in Tribolium castaneum (Herbst). Pestic. Sci. 28, 101-115.

Daglish, G.J., Nayak, M.K., Pavic, H., 2014. Phosphine resistance in Sitophilus oryzae (L.) from eastern Australia: inheritance, fitness and prevalence. J. Stored Prod. Res. 59, 237-244.

FAO, 1975. Recommended methods for detection and measurement of resistance of agricultural pests to pesticides - tentative method for adults of some major pest species of stored cereals, with methyl-bromide and phosphine - FAO method no 16. FAO Plant Prot. Bull. 23, 12-25.

GenStat 11 Committee, 2008. GenStat for Windows, Release 11.1. VSN International Ltd., Oxford, UK.

Herron, G.A., 1990. Resistance to grain protectants and phosphine in coleopterous pests of grain stored on farms in New South Wales. J. Aust. Entomol. Soc. 29, 183-189.

Hinton, H.E., 1942. Secondary sexual characters of Tribolium. Nature 149, 500-501.

Jagadeesan, R., Collins, P.J., Daglish, G.J., Ebert, P.R., Schlipalius, D.I., 2012. Phosphine resistance in the rust red flour beetle, Tribolium castaneum (Herbst) (Coleoptera: Tenebrionidae): Inheritance, gene interactions and fitness costs. PLoS One 7 (2), e31582. http://dx.doi.org/10.1371/journal.pone.0031582.

Jagadeesan, R., Fotheringham, A., Ebert, P.R., Schlipalius, D.I., 2013. Rapid genome wide mapping of phosphine resistance loci by a simple regional averaging analysis in the red flour beetle, Tribolium castaneum. BMC Genomics 14, 650.

McKenzie, J.A., 1996. Ecological and Evolutionary Aspects of Insecticide Resistance. R.G. Landes Company, Austin.

Opit, G.P., Phillips, T.W., Aikins, M.J., Hasan, M.M., 2012. Phosphine resistance in Tribolium castaneum and Rhyzopertha dominica from stored wheat in Oklahoma. J. Econ. Entomol. 105, 1107-1114.

Pimentel, M.A.G., D'A Faroni, L.R., Corrêa, A.S., Guedes, R.N.C., 2011. Phosphineinduced walking response of the lesser grain borer (Rhyzopertha dominica). Pest Manag. Sci. 68, 1368-1373.

Pimentel, M.A.G., D'A Faroni, L.R., Tótola, M.R., Guedes, R.N.C., 2007. Phosphine resistance, respiration rate and fitness consequences in stored-product insects. Pest Manag. Sci. 63, 876-881.

Price, L.A. Mills, K.A. 1988. The toxicity of phosphine to the immature stages of resistant and susceptible strains of some common stored product beetles, and implications for their control. J. Stored Prod. Res. 24, 51-59.

Rajendran, S., 1994. The current status of phosphine fumigations in India. In: Highley, E., Wright, E.J., Banks, H.J., Champ, B.R. (Eds.), Proceedings of the Sixth International Working Conference on Stored-product Protection, Canberra, Australia, April 17-23, 1994. CAB International, Wallingford, UK, pp. 148-152.

Schlipalius, D.I., Chen, W., Collins, P.J., Nguyen, T., Reilly, P.E.B., Ebert, P.E., 2008. Gene interactions constrain the course of evolution of phosphine resistance in the lesser grain borer, Rhyzopertha dominica. Heredity 100, 506-516.

Sokal, R.R., Rohlf, F.J., 1995. Biometry. W.H. Freeman, New York.

Sousa, A.H., D'A Faroni, L.R., Pimentel, M.A.G., Guedes, R.N.C., 2009. Developmental and population growth rates of phosphine-resistant and -susceptible populations of stored-product insect pests. J. Stored Prod. Res. 45, 241-246.

White, G.G., Lambkin, T.A., 1990. Baseline responses to phosphine and resistance status of stored-grain beetle pests in Queensland, Australia. J. Econ. Entomol. $83,1738-1744$. 